\title{
Drug management in older Parkinson's disease patients
}

James KH Luk, MBBS(HK), MSc (Experimental Medicine)(UBC), FRCP(Edin, Glasg, Irel), FHKCP, FHKAM (Medicine)

\begin{abstract}
Parkinson's disease (PD) is a common degenerative neurological disorder in older people. Its management is primarily focused on symptom control and maintenance of self-care and quality of life. The use of medication for PD is affected by patient age, symptoms and degree of disability, clinician experience, and drug cost, availability and side effects, as well as patient choice. This article discusses practical tips and myths of drug management for older PD patients.
\end{abstract}

\author{
Department of Medicine and Geriatrics, \\ Fung Yiu King Hospital, Pokfulam, Hong \\ Kong
}

Correspondence to: Dr James KH Luk, Department of Medicine and Geriatrics, Fung Yiu King Hospital, 9 Sandy Bay Road, Pokfulam, Hong Kong. Email: lukkh@ha.org.hk

\section{INTRODUCTION}

Parkinson's disease (PD) is a common degenerative neurological disorder in older people. Its management is primarily focused on symptom control and maintenance of self-care and quality of life (QOL). The use of medication for PD is affected by patient age, symptoms and degree of disability, clinician experience, and drug cost, availability and side effects, as well as patient choice. The range of PD medication is very broad, and drug treatment for PD is challenging to clinicians. ${ }^{1}$ This study discusses practical tips and myths of drug management for older PD patients. Readers should refer to neurology textbooks for a comprehensive review and treatment of PD.

\section{LEVODOPA AS FIRST-LINE TREATMENT}

Early use of a dopamine agonist has been advocated in newly diagnosed PD patients. ${ }^{2}$ The use of levodopa can be postponed to the later course of the disease. There is concern about levodopa neurotoxicity, as free radicals generated by the oxidative metabolism of dopamine might contribute to dopaminergic neuronal degeneration. Nonetheless, there is no evidence that levodopa is toxic in humans, although the findings of neurotoxicity in vitro and in some in vivo models has raised concern about its safety. ${ }^{3}$ In a large, multicentre, randomised, controlled trial comparing levodopa with placebo in patients with early PD (the ELLDOPA study), levodopa did not appear to be neurotoxic and may even be neuroprotective. ${ }^{4}$ It remains unclear whether levodopa can positively or negatively affect the natural history of PD. ${ }^{5}$ Compared with a dopamine agonist, levodopa results in a higher incidence of dyskinesia and motor fluctuations. ${ }^{6}$ Yet there is evidence that choice of initial therapy has little impact on such incidence. There is an unproven concept that patient responsiveness to levodopa is finite. It is uncertain whether reduction in responsiveness to levodopa over time is due to a decline in drug response or progression of disease.

In older patients, it is advocated that levodopa should be the first-line therapy, as it is the most effective drug to improve PD symptoms. ${ }^{7}$ A 'wait and watch' policy may not be appropriate in older PD patients. Few PD patients can be satisfactorily maintained on dopamine agonist monotherapy for more than a few years before levodopa is needed. Dopamine agonists are more likely than levodopa to cause hallucinations. ${ }^{8,9}$ They may also induce somnolence or sleep attacks. Severe leg oedema can occur occasionally and is difficult to treat without drug cessation. Therefore, unless contraindicated, levodopa should be started early in older PD patients to enhance their QOL.

\section{TITRATING LEVODOPA}

In elderly patients with early-stage PD, levodopa should be started at the minimal effective dose, usually 50 to $100 \mathrm{mg}$ in combination with a 
decarboxylase inhibitor three times a day. Either levodopa/carbidopa or levodopa/benserazide can be used as they are equally effective. The dosage should be increased slowly in accordance with the patient's response. An optimal effect is generally achieved with a daily dosage corresponding to $300-800 \mathrm{mg}$ levodopa divided into 3 or more doses. In older PD patients, medication should be titrated slowly and gradually, based on the principle of 'start low and go slow'. The treatment target is not complete resolution of PD symptoms, but rather reasonable QOL and mobility.

\section{PREPARATIONS OF LEVODOPA}

Levodopa has to be taken orally as absorption takes place in the small intestine. ${ }^{10}$ Standard levodopa tablets are breakable and can be ground to facilitate swallowing. Nonetheless, ground levodopa tablets are usually bitter and may be intolerable. When taking a capsular or controlled release form of levodopa, patients have to swallow the whole drug without chewing. There is no liquid form or sublingual preparation. In some countries, orally soluble carbidopa/levodopa preparations are available in which the medications can dissolve on the tongue. They are particularly good for dysphagic patients who cannot swallow the whole tablet. In Hong Kong, dispersible levodopa/benserazide is available and can be dissolved in a glass of water $(25-50 \mathrm{ml})$. The tablets disintegrate completely within a few minutes, producing a milky-white dispersion. This is easier to be administered to dysphagic patients or patients on tube feeding, and achieves a faster drug response. ${ }^{11}$ Because of rapid sedimentation, it is advisable to stir the dispersion before drinking. The dispersible tablets should be taken within 30 minutes of preparation.

Controlled-release preparations of levodopa, $100 \mathrm{mg}$ or $200 \mathrm{mg}$, have few advantages over shorter-acting preparations apart from their use in treating nocturnal disabilities. These preparations have reduced bioavailability (as low as 50\% to $70 \%$ of the short-acting preparations), and patients often complain of their slow onset of action. ${ }^{12}$ Both shorter-acting and controlled-release preparations are used in some situations to bring a faster drug response, but the effect is often unpredictable. They are prevented from entering the circulation when the stomach is empty, and do not cross the blood-brain barrier when taken with or after meals. ${ }^{13}$ The optimal dose of the sustained-release formulation to produce the most consistent clinical response has not been studied. In addition, controlled-release preparations cannot be crushed and thus are difficult to swallow for dysphagic patients.

When food is taken with levodopa, protein in food can interfere with absorption of levodopa into the bloodstream. Additionally, high blood levels of protein can interfere with transport of levodopa via the blood brain barrier. One group of amino acids-large neutral amino acids-can compete with levodopa and prevent it from entering the brain where it is turned into dopamine. ${ }^{14}$ Therefore, levodopa is best taken at least 30 minutes before or 60 minutes after a meal. Altering protein in the diet may be necessary in advanced PD patients in whom motor fluctuations are prominent. People who experience motor fluctuations can benefit from regulating the protein in their diet. ${ }^{15}$ In some patients, particularly those who are first started with levodopa, undesirable gastrointestinal effects can occur. This can be reduced by taking levodopa with a small low protein snack. If the symptom persists, domperidone may be helpful.

\section{DOPAMINE AGONIST}

Non-ergoline-derived dopamine agonists are available. Ergoline-derived dopamine agonists are rarely used today due to the risk of peritoneal, pulmonary, and valvular fibrosis. ${ }^{16}$ Most dopamine agonists have to be taken orally, except rotigotine that can be delivered transdermally. ${ }^{17}$ Transdermal administration avoids interaction with food and first past effect, has a sustained-release, and requires only once-a-day application. It can be given easily in patients with severe dysphagia or those who temporarily cannot tolerate oral medications. The drawbacks of this drug are skin allergy, peripheral oedema, increased hallucination, and cost.

\section{MORNING AKINESIA}

When the next levodopa dose produces a delayed onset of action, it is called delayed 'on'. Delayed 'on' of the first levodopa daily dose results in morning akinesia, which can significantly affect QOL and impair daily activities. ${ }^{18} \mathrm{PD}$ patients often experience difficulty in swallowing the first dose as they are in 
the 'off' state. Dispersible levodopa is easier to be taken by older patients in an 'off' state and has a faster action. In the AM IMPAKT trial, subcutaneous injection of apomorphine reduces the time to 'on' in PD patients and is generally well tolerated. ${ }^{19} \mathrm{In}$ addition, sublingual apomorphine appears to be a convenient, rapid, and reliable method for treating 'off' episodes. ${ }^{20}$ Nonetheless, only a subcutaneous form of apomorphine is currently available in Hong Kong.

\section{WITHDRAWAL OF LEVODOPA}

People with PD have limited brain reserves of endogenous dopamine. Omission or delay in levodopa or dopamine agonist administration leads to a significant drop in brain dopamine levels. This may lead to deterioration in disease control, with distressing symptoms such as tremor, pain, rigidity, dysphagia, and immobility. Abrupt withdrawal of levodopa may also result in a neuroleptic malignantlike syndrome. ${ }^{21}$ Common reasons for being unable to take oral medications are neurogenic dysphagia or concurrent illness, gastrointestinal bleeding, gastroenteritis, iatrogenic 'nil by mouth' status especially perioperatively, and impaired consciousness. In these situations, administration of drug via an enteral tube or use of transdermal rotigotine can maintain a continuous dopaminergic activity in the patients. Subcutaneous apomorphine can also be used with an equivalent dose of levodopa to apomorphine calculated. ${ }^{22}$ Specialist advice is suggested if apomorphine is used.

\section{SUDDEN SLEEP}

Both dopamine agonists and levodopa have been associated with somnolence and episodes of sudden sleep onset. ${ }^{23}$ Sudden onset of sleep during meals or watching TV has been reported in older PD patients prescribed dopamine agonists or levodopa. Older patients may suffer from falls during these episodes. Patients and carers must be informed of this and advised to exercise caution.

\section{ENTERAL FEEDING}

The prevalence of oropharyngeal dysphagia in patients with PD can be as high as $81 \% .{ }^{24}$ Many older patients with advanced PD require enteral feeding, such as nasogastric tube and percutaneous endoscopic gastrostomy with or without jejunal extension. ${ }^{25}$ Special precautions are needed in administrating oral drugs to reduce the chance of blockage. Tablets should be replaced by syrup or transdermal preparations, if available. Tablets that can be dissolved in water such as the dispersible form should be used. If unavailable, tablets must be crushed and mixed with water. A sieve may be used to filter out un-dissolved particles. This is particularly important if drugs are administered via a small calibre enteral tube. The tube must be flushed before and after each medicine. If more than one medicine is being given, the tube should be flushed between each. In case of percutaneous endoscopic gastrostomy with jejunal extension, medications have to be given via the gastric tube instead of the small bore jejunal tube.

\section{MONOAMINE OXIDASE ISOENZYME TYPE B INHIBITORS}

Non-specific monoamine oxidase (MAO) isoenzyme inhibitors can extend the availability of dopamine and relieve PD symptoms. However, their interaction with tyramine-containing foods may lead to hypertensive crisis. In addition, they may interact with levodopa, dopamine agonists, and many other anti-PD medications. The development of MAO-B inhibitors enables the use of this group of drugs in PD patients without significant interactions with food and other drugs. ${ }^{26}$ Two MAO-Bs are available, namely selegiline and rasagiline. The latter is more potent, has fewer side effects, and may be more neuroprotective in PD patients than selegiline. Nevertheless, the two drugs are comparable in terms of efficacy and side-effect profile. ${ }^{27}$ The claim of neuroprotection is limited to animal studies and has not been proven in humans. ${ }^{28}$ When applied as monotherapy, $\mathrm{MAO}-\mathrm{B}$ inhibitors provide a modest improvement in motor function and delay the need for levodopa. In older PD patients, MAO-B inhibitors are often used as add-on to levodopa as they can significantly reduce the off-time.

\section{AMANTADINE}

Amandatine is an old anti-viral drug that was coincidentally found to ameliorate PD symptoms. Nonetheless, it has many side effects including dizziness, anorexia, and blurred vision. It also leads to confusion and hallucination in older patients. Toxic 
levels of amantadine can occur in older patients with renal impairment. Withdrawal of the drug may lead to delirium, neuroleptic malignant syndrome, and motor deterioration..$^{29,30}$ Hence, it is not commonly used in older patients with PD, particularly those with cognitive impairment. Nonetheless, amantadine can reduce levodopa-induced dyskinesia and may have a role in selected PD patients with such condition. ${ }^{31,32}$

\section{ANTI-CHOLINERGIC AGENTS}

Drugs like benzhexol and benztropine are useful adjuncts to levodopa in helping to control resting tremor and dystonia. They may also improve drooling. ${ }^{33}$ They are best avoided in elderly patients as they may lead to urinary retention, confusion with hallucination, constipation, blurred vision, dry mouth, and precipitation of narrow angle glaucoma. Anticholinergic drugs are associated with reduced functional outcome in older patients during rehabilitation. ${ }^{34}$ Disturbing neuropsychiatric symptoms are often an indication to withdraw anticholinergic agents. This is best done in a gradual manner to avoid withdrawal symptoms.

\section{DOMPERIDONE}

Delayed gastric emptying is a common problem in PD. Metoclopamide should not be used as it crosses the blood-brain barrier and worsens PD symptoms by blocking the D2 dopamine receptors. ${ }^{35}$ Domperidone, a dopamine antagonist that does not easily cross the blood-brain barrier, is suitable for treating gastrointestinal symptoms in PD patients because of minimal extrapyramidal adverse effects. Low-dose domperidone slightly increases the initial bioavailability of levodopa and may increase its initial rate of absorption. ${ }^{36}$ There is a concern about domperidone-associated cardiotoxicity in older PD patients. ${ }^{37}$ At this moment, no definitive conclusions can be drawn about its safety in older patients. Although domperidone is the first choice for treating gastrointestinal symptoms associated with $\mathrm{PD}$, monitoring for potential cardiotoxic effects is necessary when doses above $30 \mathrm{mg}$ per day are prescribed.

\section{HALLUCINATION, DEMENTIA, AND DEPRESSION}

Hallucination and other psychotic features such as delusion and paranoia may develop in older PD patients on treatment. ${ }^{38}$ These may be due to levodopa side effects, underlying dementia prior to recognition of PD, development of dementia after PD, or dementia with Lewy bodies (dementia prior to or within 12 months of development of PD). ${ }^{39}$ Hallucinations in PD patients are typically visual. ${ }^{40}$ They are generally complex and formed, in the form of people or animals. The hallucinations tend to be fixed. Older PD patients may report seeing vivid images several times a week such as helicopters spying on them or fire in their home. Seeing tiny creatures such as ants or cockroaches is not uncommon. Psychiatric side effects are often difficult to manage. For psychiatric problems that are caused by anti-PD therapy, some improvement can be achieved by reducing or terminating treatment. The strategy is a sequence of drug withdrawal in the following order: amantadine/ selegiline, anticholinergics, dopamine agonists, catechol-O-methyltransferas inhibitor and finally levodopa ${ }^{41}$ The use of cholinesterase inhibitors can be considered. ${ }^{42,43}$ Rivastigmine, oral or transdermal, may improve cognitive function and psychotic features of PD patients. ${ }^{44}$ Although there is concern about worsening of PD symptoms with increased cholinergic activity when cholinesterase inhibitors are given, rivastigmine treatment does not worsen motor symptoms in PD patients.Typical anti-psychotic drugs, such as haloperidol, which antagonise dopamine D2 receptors, may induce exacerbation of $\mathrm{PD}$ and should not be used. If necessary, clozapine or quetiapine can be considered as they appear to control psychosis without comprising motor function. ${ }^{45}$ As clozapine has been related to agranulocytosis, quetiapine is usually preferred in older patients.

Depression is common in PD patients. ${ }^{46}$ Although it can be a reaction to the disease, there is also evidence that depression is endogenous and intrinsic to the condition as part of the neurochemical profile. Both selective serotonin reuptake inhibitors and serotonin with norepinephrine reuptake inhibitors are effective in treating depression among $\mathrm{PD}$ patients without worsening the motor symptoms. ${ }^{47}$ Tricylic anti-depressants are also effective, but they are not a good choice for older PD patients because of their numerous side effects.

\section{CONCLUSION}

Clinicians of older PD patients should have good 
knowledge of different PD medications. Older patients with difficult-to-control PD symptoms are best managed by a multidisciplinary team led by geriatricians, neurologists, or rehabilitation specialists. Comprehensive geriatric assessment is needed to address social issues, carer stress, feeding problems, advance care planning, and end-of-life care matters. ${ }^{48}$ Geriatric rehabilitation should be provided when appropriate to foster mobility and functional state, with the aim of maximising QOL in older PD patients. ${ }^{49}$

\section{REFERENCES}

1. Chan DK. The art of treating Parkinson disease in the older patient. Aust Fam Physician 2003;32:927-31.

2. Bonuccelli U, Del Dotto P, Rascol O. Role of dopamine receptor agonists in the treatment of early Parkinson's disease. Parkinsonism Relat Disord 2009;15(Suppl 4):S44-53.

3. Fahn S. Levodopa-induced neurotoxicity: does it represent a problem for the treatment of Parkinson's disease? CNS Drugs 1997;8:376-93.

4. Fahn S, Oakes D, Shoulson I, et al. Levodopa and the progression of Parkinson's disease. N Engl J Med 2004;351:2498-508.

5. ValeoT. Is levodopa neurotoxic? New data — and the controversy lives on. Neurol Today 2011;11:132-4.

6. van Laar T. Levodopa-induced response fluctuations in patients with Parkinson's disease: strategies for management. CNS Drugs 2003;17:475-89.

7. Grosset D, Taurah L, Burn DJ, et al. A multicentre longitudinal observational study of changes in self reported health status in people with Parkinson's disease left untreated at diagnosis. J Neurol Neurosurg Psychiatry 2007;78:465-9.

8. Rascol O, Brooks DJ, Korczyn AD, et al. A five-year study of the incidence of dyskinesia in patients with early Parkinson's disease who were treated with ropinirole or levodopa. N Engl J Med 2000;342:1484-91.

9. Holloway RG, Shoulson I, Fahn S, et al. Pramipexole vs levodopa as initial treatment for Parkinson disease: a 4-year randomized controlled trial. Arch Neurol 2004;61:1044-53.

10. Camargo SM,Vuille-dit-Bille RN, Mariotta L, et al. The molecular mechanism of intestinal levodopa absorption and its possible implications for the treatment of Parkinson's disease. J Pharmacol Exp Ther 2014;351:114-23.

11. Jansson Y, Eriksson B, Johnels B. Dispersible levodopa has a fast and more reproducible onset of action than the conventional preparation in Parkinson's disease. A study with optoelectronic movement analysis. Parkinsonism Relat Disord 1998;4:201-6.

12. LeWitt PA. Clinical studies with and pharmacokinetic considerations of sustained-release levodopa. Neurology 1992;42(1 Suppl 1):S29-60.

13. Yeh KC, August TF, Bush DF, et al. Pharmacokinetics and bioavailability of Sinemet CR: a summary of human studies. Neurology 1989;39:25-38.

14. Robertson DR, Higginson I, Macklin BS, Renwick AG, Waller DG, George CF. The influence of protein containing meals on the pharmacokinetics of levodopa in healthy volunteers. Br J Clin Pharmacol 1991;31:413-7.

15. Barichella M, Marczewska A, De Notaris R, et al. Special lowprotein foods ameliorate postprandial off in patients with advanced Parkinson's disease. Mov Disord 2006;21:1682-7.

16. Borovac JA. Side effects of a dopamine agonist therapy for Parkinson's disease: a mini-review of clinical pharmacology. Yale
J Biol Med 2016;89:37-47.

17. Bunten S, Happe S. Rotigotine transdermal system: a short review. Neuropsychiatr Dis Treat 2006;2:421-6.

18. Tambasco N, Simoni S, Nigro P, Paolini Paoletti F, Marsili E, Calabresi P. Morning akinesia in Parkinson's disease: challenges and solutions. J Parkinsonism Restless Legs Syndr 2016;6:57-63.

19. Isaacson S, Lew M, Ondo W, Hubble J, Clinch T, Pagan F. Apomorphine subcutaneous injection for the management of morning akinesia in Parkinson's disease. Mov Disord Clin Pract 2017;4:78-83.

20. Hauser RA, Olanow CW, Dzyngel B, et al. Sublingual apomorphine (APL-130277) for the acute conversion of OFF to ON in Parkinson's disease. Mov Disord 2016;31:1366-72.

21. Man SP. An uncommon adverse effect of levodopa withdrawal in a patient taking antipsychotic medication: neuroleptic malignant-like syndrome. Hong Kong Med J 2011;17:74-6.

22. Haq IU, Lewitt PA, Fernandez HH. Apomorphine therapy in Parkinson's disease: a review. Expert Opin Pharmacother 2007;8:2799-809.

23. Paus S, Brecht HM, Köster J, Seeger G, Klockgether T, Wüllner $\mathrm{U}$. Sleep attacks, daytime sleepiness, and dopamine agonists in Parkinson's disease. Mov Disord 2003;18:659-67.

24. Takizawa C, Gemmell E, Kenworthy J, Speyer R. A systematic review of the prevalence of oropharyngeal dysphagia in stroke, Parkinson's disease, Alzheimer's disease, head injury, and pneumonia. Dysphagia 2016;31:434-41.

25. Lin OY, Luk JK, Ng MM, Chan FH. Jejunal feeding in an elderly man suffering from advanced Parkinson's disease. Asian J Gerontol Geriatr 2013;8:50-3.

26. Dezsi L, Vecsei L. Monoamine oxidase B inhibitors in Parkinson's disease. CNS Neurol Disord Drug Targets 2017;16:425-39.

27. Cereda E, Cilia R, Canesi M, et al. Efficacy of rasagiline and selegiline in Parkinson's disease: a head-to-head 3-year retrospective case-control study. J Neurol 2017;264:1254-63.

28. Kong P, Zhang B, Lei P, et al. Neuroprotection of MAO-B inhibitor and dopamine agonist in Parkinson disease. Int J Clin Exp Med 2015;8:431-9.

29. Fryml LD, Williams KR, Pelic CG, et al. The role of amantadine withdrawal in 3 cases of treatment-refractory altered mental status. J Psychiatr Pract 2017;23:191-9.

30. Marxreiter F, Winkler J, Uhl M, Madžar D. A case report of severe delirium after amantadine withdrawal. Case Rep Neurol 2017;9:44-8.

31. Luginger E, Wenning GK, Bösch S, Poewe W. Beneficial effects of amantadine on L-dopa-induced dyskinesias in Parkinson's disease. Mov Disord 2000;15:873-8.

32. Oertel W, Eggert K, Pahwa R, et al. Randomized, placebocontrolled trial of ADS-5102 (amantadine) extended-release capsules for levodopa-induced dyskinesia in Parkinson's disease (EASE LID 3). Mov Disord 2017. [Epub ahead of print].

33. Ou R, Guo X, Wei Q, et al. Prevalence and clinical correlates of drooling in Parkinson disease: a study on 518 Chinese patients. Parkinsonism Relat Disord 2015;21:211-5.

34. Koshoedo S, Soiza RL, Purkayastha R, Mangoni AA. Anticholinergic drugs and functional outcomes in older patients undergoing orthopaedic rehabilitation. Am J Geriatr Pharmacother 2012;10:251-7.

35. López-Sendón J, Mena MA, de Yébenes JG. Drug-induced parkinsonism. Expert Opin Drug Saf 2013;12:487-96.

36. Nishikawa $N$, Nagai $M$, Tsujii T, Iwaki $H$, Yabe $H$, Nomoto $M$. Coadministration of domperidone increases plasma levodopa concentration in patients with Parkinson disease. Clin Neuropharmacol 2012;35:182-4.

37. Lertxundi U, Domingo-Echaburu S, Soraluce A, García M, RuizOsante B, Aguirre C. Domperidone in Parkinson's disease: a perilous arrhythmogenic or the gold standard? Curr Drug Saf 2013;8:63-8. 
38. Fénelon G. Psychosis in Parkinson's disease: phenomenology, frequency, risk factors, and current understanding of pathophysiologic mechanisms. CNS Spectr 2008;13(3 Suppl 4):18-25.

39. Hanagasi HA, Tufekcioglu Z, Emre M. Dementia in Parkinson's disease. J Neurol Sci 2017;374:26-31.

40. Frei K, Truong DD. Hallucinations and the spectrum of psychosis in Parkinson's disease. J Neurol Sci 2017;374:56-62.

41. Kuzuhara S. Drug-induced psychotic symptoms in Parkinson's disease. Problems, management and dilemma. J Neurol 2001;248(Suppl 3):III28-31.

42. O'Brien JT, Holmes C, Jones M, et al. Clinical practice with antidementia drugs: a revised (third) consensus statement from the British Association for Psychopharmacology. J Psychopharmacol 2017;31:147-68.

43. Rolinski M, Fox C, Maidment I, McShane R. Cholinesterase inhibitors for dementia with Lewy bodies, Parkinson's disease dementia and cognitive impairment in Parkinson's disease. Cochrane Database Syst Rev 2012;3:CD006504.
44. Cummings JL. Cholinesterase inhibitors for treatment of dementia associated with Parkinson's disease. J Neurol Neurosurg Psychiatry 2005;76:903-4.

45. Rabey JM, Prokhorov T, Miniovitz A, Dobronevsky E, Klein C. Effect of quetiapine in psychotic Parkinson's disease patients: a double-blind labeled study of 3 months' duration. Mov Disord 2007;22:313-8.

46. Goodarzi Z, Mrklas KJ, Roberts DJ, Jette N, Pringsheim T, Holroyd-Leduc J. Detecting depression in Parkinson disease: a systematic review and meta-analysis. Neurology 2016;87:42637.

47. Haasum Y, Fastbom J, Johnell K. Use of antidepressants in Parkinson's disease: a Swedish register-based study of over 1.5 million older people. Parkinsonism Relat Disord 2016;27:85-8.

48. Luk JK, Or KH, Woo J. Using the comprehensive geriatric assessment technique to assess elderly patients. Hong Kong Med J 2000;6:93-8.

49. Luk JK. Rehabilitation in older people: know more, gain more. Hong Kong Med J 2012;18:56-9. 